\title{
Change of Antigenic Determinants of SARS-CoV-2 Virus S-Protein as a Possible Cause of Antibody-Dependent Enhancement of Virus Infection and Cytokine Storm
}

\author{
Yu. D. Nechipurenko ${ }^{a, *}$, A. A. Anashkina ${ }^{a}$, and O. V. Matveeva ${ }^{b, c}$ \\ ${ }^{a}$ Engelhardt Institute of Molecular Biology, Russian Academy of Sciences, Moscow, 119991 Russia \\ ${ }^{b}$ Sendai Viralytics LLC, Acton, MA 01720 USA \\ ${ }^{c}$ Biopolymer Design LLC, Acton, MA 01721 USA \\ *e-mail: nech99@mail.ru
}

Received May 28, 2020; revised May 28, 2020; accepted June 5, 2020

\begin{abstract}
A hypothesis is proposed that the cytokine storm syndrome, which complicates COVID-19 in some patients, is a consequence of antibody-dependent enhancement of virus infection, which is in turn happens due to a change in dominant antigenic determinants of SARS-CoV-2 S-protein. The antibody-dependent enhancement of virus infection is a phenomenon in which virus-specific antibodies that are not neutralizing enhance the entry of infectious virus into immune cells causing their death. Antibody-dependent enhancement has been reported for different coronaviruses. This phenomenon happens due to a decrease in the binding strength of neutralizing antibodies to the virus, which converts these antibodies into suboptimalnot neutralizing ones. According to our hypothesis, such a decrease in affinity may be associated with a change in the conformation of the viral S-protein. We believe that this conformational change is the major factor in the switching of antibodies affinity, which triggers antibody-dependent enhancement. However, other factors that contribute to antigen drift and antigenic determinant changes may also play a role.
\end{abstract}

Keywords: COVID-19, coronavirus, SARS-CoV-2, antigen-dependent enhancement of infection, ADE, spike protein, S-protein, change in conformations, antigenic determinants, change in epitopes

DOI: $10.1134 / \mathrm{S} 0006350920040119$

\section{INTRODUCTION}

The SARS-CoV-1 and SARS-CoV-2 viruses infect host cells via the ACE2 receptor [1]. However, the SARS-CoV-1 can enter cells that are lacking this receptor using alternative cell entry strategy that is called antibody-dependent enhancement of viral infection (ADE) [2-4]. ADE phenomenon is described for different viruses but it is more common for viruses, which genomes are represented by the $(+)$ RNA strand [5], including coronaviruses [1, 2, 7-9].

\section{ANTIBODY DEPENDENT DISEASE ENHANCEMENT}

Figure 1 shows a scheme that visualizes ADE phenomenon for coronaviruses (right) by contrasting it with an efficient immune response that promotes virus clearance (left). Both types of responses involve complex formation of IgG antibodies with a virus that via FcyII receptors can be absorbed by CD32+ immune cells $[6,8,10-13]$. However, this process might lead to the destruction of a virus inside an immune cell [10] or to intracellular viral replication [2, 3,6-8]. During the process that results in efficient immune response, the virus cannot escape a complex that is formed with high affinity neutralizing antibodies - and is getting destroyed by cellular proteases and RNases [10]. Thus, this type of $\mathrm{IgG}$-virus complex formation and the immune cell absorption leads to the virus clearance that can be followed by a patient recovery as shown in the left side of Fig. 1. Alternatively, the virus escapes the IgG-virus complex inside the immune cell and begins a replication cycle casing ADE. This happens if the virus forms complex with low affinity non-neutralizing IgG antibodies, as shown in the right side of the Fig. 1. The virus replication in immune cell can be abortive without production of any viable virions. Nevertheless, even abortive virus infection can lead to mass death of immune cells, which in turn can cause inflammation cascade and a cytokine storm. CD32+ immune cells include monocytes, macrophages, some categories of B cells, and some dendritic cells.

ADE was demonstrated for SARS-CoV-1 and other coronaviruses [2-4,6-8]. So far it was not shown for SARS-CoV-2. However, recent publications and preprints indicate that SARS-CoV-2 can infect human primary CD4+ $\mathrm{T}$ cells [11] and some other immune cells [12]. This non-productive or weak 


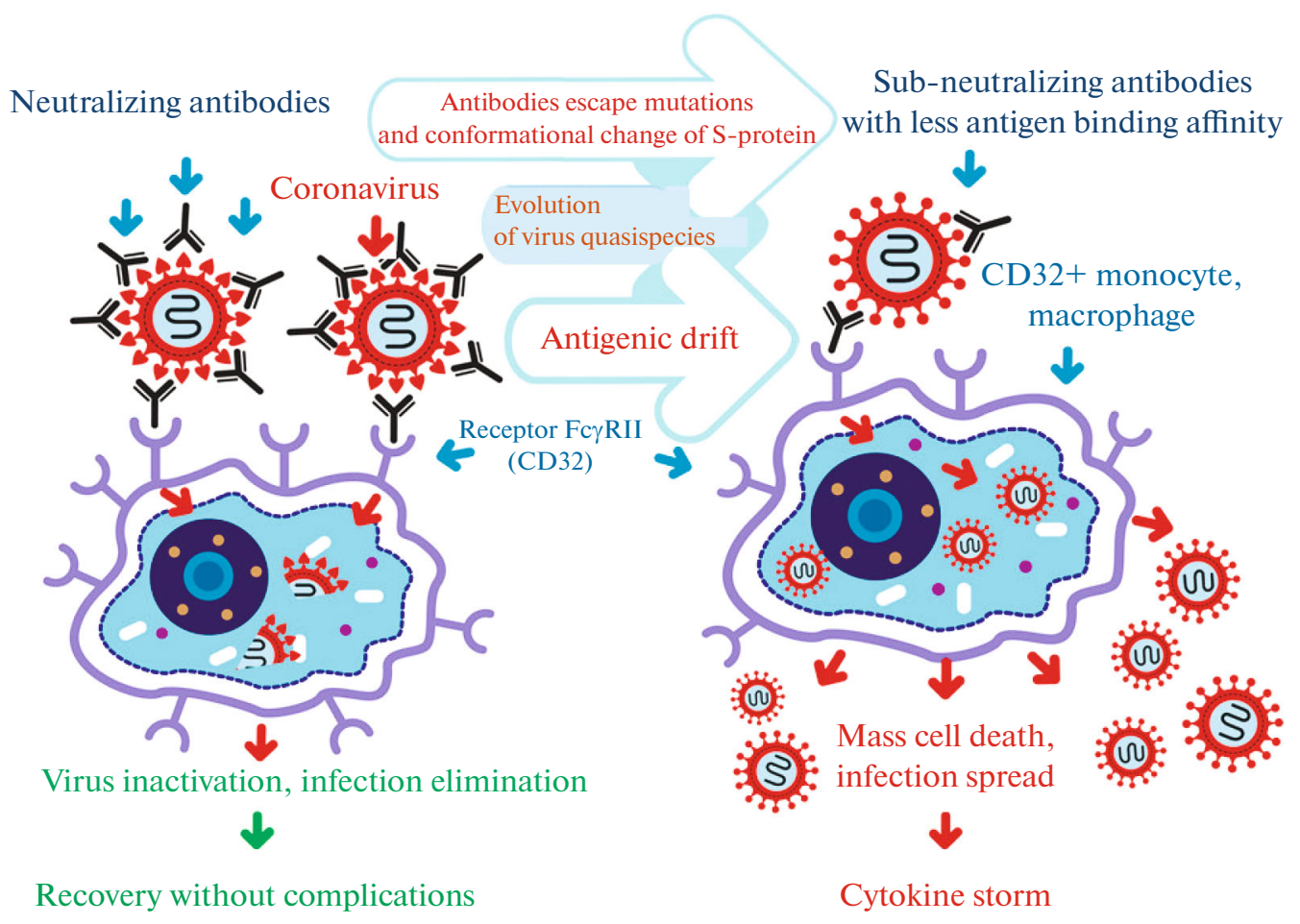

This graphic illustration was designed and created by Olga Zolotuchina, Olga Matveeva and Yurii Nechipurenko

Fig. 1. Antibody-dependent enhancement of viral infection.

low titer infection is unrelated to ADE. Perhaps it can be further enhanced by $\operatorname{IgG}$ antibodies targeting S-protein.

The family of Fc receptors for IgG antibody binding is broadly expressed by variable immune cells. However, only a subset of these cells expresses FcyII receptor that can be involved in ADE process [13]. The functional categorization of antibodies, which include the ADE causing category, was proposed in [14].

According to some researchers, monocytes and macrophages play a key role in the acute inflammation process that occurs in some COVID-19 patients [15]. These observations can be explained if we assume that the virus acquires, due to ADE, the ability to infect these immune cells. The concern about potential danger of ADE for COVID-19 pathogenesis was formulated by some researches [16, 17]. Perhaps beginning of SARS-CoV-2 replication in immune cells is a key step in the development of the disease and its evolution from mild to severe form. ADE can explain the observed dysregulation of the immune system, including mass apoptosis of immune cells, as well as a development of cytokine storm in some patients.

Some immunodominant antigenic determinants of the receptor binding domain (RBD) of S-protein of the SARS-CoV-1 and MERS-CoV viruses cause ADE $[3,8]$. Is it possible that similar determinants of
SARS-CoV-2 are doing the same? We will look at this issue in the following sections.

\section{CHARACTERISTICS OF THE SPIKE PROTEIN OF THE SARS-CoV-1 AND SARS-CoV-2 VIRUSES}

Coronavirus Spike protein (S-protein) forms "spikes" of viral "crown" that are the characteristic structures of the virus family. The S-proteins of SARS-CoV- 1 and SARS-CoV-2 viruses are very similar in terms of amino acid sequence and in terms of function. In both viruses the S-protein is responsible for binding of ACE2 cell receptor via RBD domain receptor $[1,18-21]$. The sequences of this domain are highly homologous between SARS-CoV-1 and SARSCoV-2 [21].

The S-protein undergoes several significant transformations during maturation and functioning, which are accompanied by conformational changes in the protein. These changes in various coronaviruses have been the subject of more than a decade of research [18, 19, 22, 23]. The S-protein transformation during the virus infection cycle includes the following steps: 1. Proteolytic cleavage of the site between the S1 and S2 subunits of the S-protein, 2. Complex formation of RBD with a cell receptor, 3 . Merging of a viral envelope and a cell membrane mediated by S-protein 

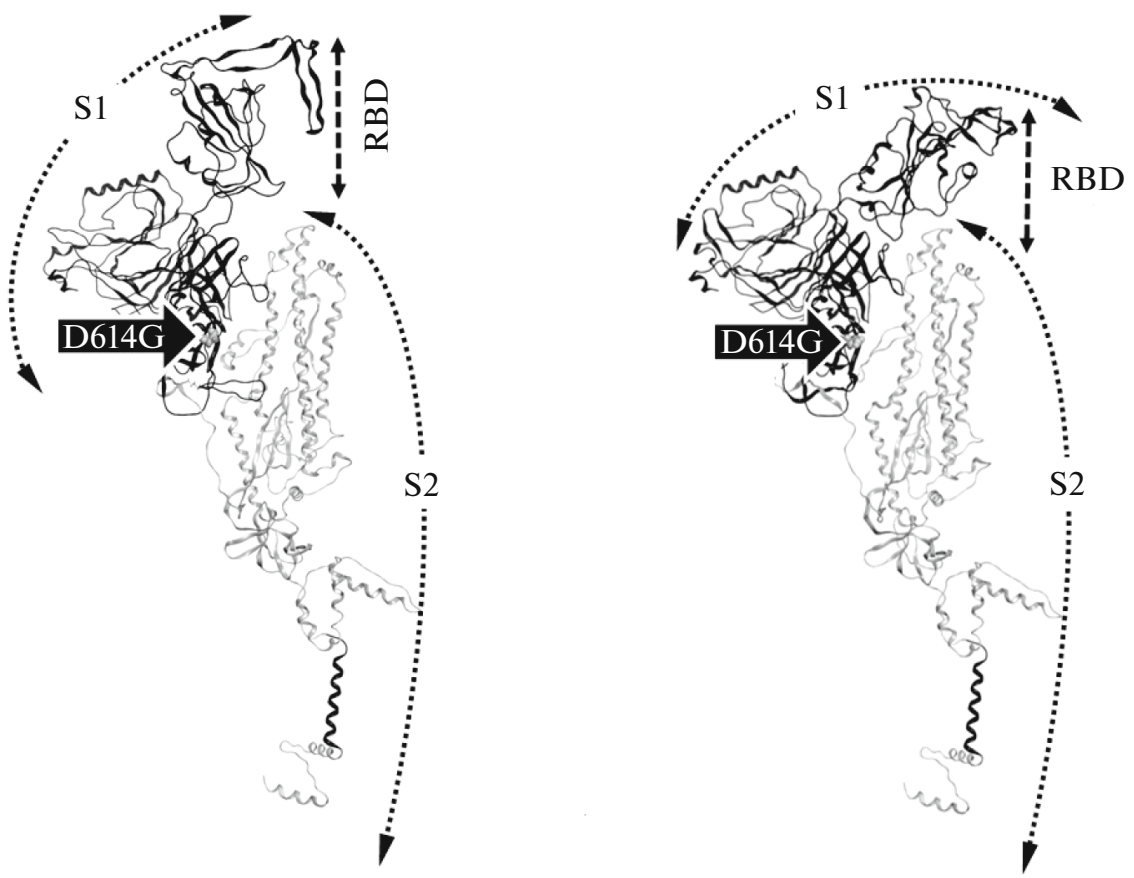

Fig. 2. Two conformations of S-protein. The spikes of SARS-CoV-2 are composed of trimers of S-protein. Each trimer protomer consists of two subunits, S1 and S2. The S1 subunit can be in two conformations: open and closed. An open conformation of S1 subunit with a raised receptor binding domain (RBD) is shown on the left. The closed conformation of this subunit with the RBD in the lowered position is shown on the right.

fusion peptide, 4. Penetration of the viral genetic material into the cell. These steps are described in some detail in a series of works. We will be interested only in certain stages of S-protein functioning for SARS-CoV-2, namely proteolytic processing, binding to the receptor, and binding to antibodies. These stages are described in detail in published works [24, 25].

The SARS-CoV-2 virus S-protein functions as a trimer; it consists of three identical molecules, which are encoded by the same gene. Each of these molecules has two subunits, namely S1 and S2 [25]. The S1 subunit can be in two conformations-open and closed, therefore RBD domain can be in up or down positions. It has been shown that the RBD domain of the SARS-CoV-2 virus S-protein is mainly in down position [1]. The form of protein with closed conformation is weakly immunogenic [26]. After approaching the ACE2 receptor, the RBD domain of SARS$\mathrm{CoV}-2$ is getting activated and binds the receptor. This interaction is characterized by a higher binding constant compared to the SARS-CoV-1 virus [26]. Open $\mathrm{S}$-protein conformation is more characteristic for the SARS-CoV-1 and closed for SARS-CoV-2 [1].

Thus, S1 subunit of the S-protein is capable to significant conformational rearrangements and can exist in at least two conformational states (see Fig. 2). Note that a biophysical study of SARS-CoV-2 S-protein and structural analysis with a resolution of $3.5 \AA$ showed that most frequently two of the three S1 sub- units of the S-protein trimer are in the closed conformation and one in the open [25]. In contrast, similar study for SARS-CoV-1 shows that the most common trimer is in which two subunits are in open conformation and one in closed [27].

If all three trimer's chains are equivalent, four different conformations are possible for a trimer. The trimetric S-protein of SARS-CoV-2 is processed by two serine proteases, one of them is represented by furin [1] and the other is TMPRSS2. Both proteases have their cleavage sites in a region at the S1/S2 boundary. These proteases break S1-S2 covalent bonds and stabilize the S1 open conformation. Partial cleavage of one or two subunits in a trimer, instead of all three, may contribute to a variety of $\mathrm{S} 1$ conformations and RBD states in a trimer.

\section{HYPOTHETICAL RELATIONSHIP BETWEEN MOLECULAR CHARACTERISTICS OF THE S-PROTEIN AND COVID-19 PATHOGENESIS \\ Possible Causal Relationship between the Change of $S$-Protein Conformations and $A D E$}

We hypothesized that antibodies developed for the SARS-CoV-2 variant of the virus with one conformation of S-protein may lose their ability to neutralize the virus when the conformation of this protein changes and, as a result, cause ADE. Our hypothesis is 
consistent with a scenario that occurs with SARS$\mathrm{CoV}-1$. Some antibodies to S-protein form complex with a virus that becomes a "Trojan horse," helping the virus to enter the host's monocytes or macrophages and start the infection process $[2,7]$.

Amino acid substitutions in RBD domain of SARS-CoV-1 can cause ADE via neutralizing antibodies that were developed to another virus variant [29]. Perhaps similar substitutions in RBD of SARSCoV-2 that were recently revealed [26] could cause some decrease in binding affinity to both the ACE2 receptor and to some neutralizing antibodies-and, consequently, cause ADE.

The ADE phenomenon is characterized in a recently published work for S-protein of MERS-CoV [8]. It was shown that RBD-specific monoclonal neutralizing antibodies can mediate the penetration of the virus into immune cells by functionally mimicking virus-specific receptors. The authors believe that antibodies targeted other sites of the S-protein that do not associate with its conformational changes are less likely to trigger ADE.

Apparently, there should be a certain range of binding constants, or binding energies, in which ADE can occur. If the binding between the virion and antibodies is strong enough, then the virus will not be able to escape inside the immune cell and will be destroyed. If the binding is too weak, then the antibody will not be able to "drag" the virus into the immune cell. ADE occurs when the binding is strong enough to "pull in" the virus into the immune cell, but not strong enough for the cell to destroy it in a complex with antibodies.

Functionally similar proteins exist in other viruses; in particular, Env protein of HIV, which is like the S-protein of coronavirus, consists of two subunits and forms a trimer [30, 31]. Interestingly, conformational changes of the trimer are not always described by individual stages of molecular states. Sometimes they are described as "transitional" states, therefore protein conformational dynamics is crucial for recognizing a receptor or an antibody [32]. One domain of the Env protein resembles the $\mathrm{S} 1$ subunit of coronavirus $\mathrm{S}$-protein. During the HIV infection, this protein domain mutates, and mutations cause changes of the virus tropism. Due to new mutations the virus gains an ability to bind new receptors and infect new cells. The studies of spike-like proteins of different viruses, which have been ongoing for decades, can help to understand the structure and dynamics of the conformational transformations of SARS-CoV-2 S-protein.

There is evidence that only SARS-CoV-2 with open RBD promotes high production of neutralizing antibodies, while the virus with closed RBD is poorly immunogenic [1]. Thus, it seems that the antigenic determinants of S-protein can change along with conformation changes of the protein.

Consequently, open conformation of S-protein can promote rapid elimination of the virus by antibodies, and closed conformation can promote ADE via infection of monocytes, macrophages, and dendritic cells. Figure 3 illustrates our hypothesis.

\section{Possible Relationship between D614G Amino Acid Substitution and $A D E$}

Some immunodominant antigenic determinants of the S-protein of the SARS-CoV-2 virus are in S1 subunit $[33,34]$. In different SARS-CoV-2 isolates, substitutions are often observed in this subunit at position 614: from aspartic acid to glycine and vice versa (D614G) [35]. It should be noted that aspartic acid is located at the border of the beta structure and the polyproline II left helix [36]. Although this amino acid position is at a certain distance from the RBD domain, it can be assumed that its replacement will lead to a change in the general conformation of the S-protein and a consequent change in antigenic determinants (as it is observed in allosteric proteins). Thus, amino acid at position 614 may be responsible for switching of the antigenic determinant from visible to invisible for immune system.

The variability of the D614G amino acid in the S-protein and its possible relationship with the COVID-19 pathogenesis were noted by researchers from Los Alamos [35]. They formulated two hypotheses. According to the first, D614G affects the immunogenic epitope of the virus directly. Thus, amino acid replacement can confer resistance to acquired coronavirus immunity, helping the virus to escape from neutralizing antibodies and to reduce the affinity of the antibody-virus complex. The second hypothesis suggests that the mutation affects another epitope involved in ADE via S-protein conformation change. In both cases, D614G substitution can enhance ADE [35].

Perhaps D614G amino acid substitution from one to another can lead to improved or, conversely, difficult access of proteases to the sites of proteolytic processing of S-protein. Some researchers believe that effective protease cleavage between the S1 and S2 subunits may contribute to the predominance of the open S-protein conformation [1]. Thus, by modulating protease access, an amino acid at a specific position of the S-protein can contribute to the predominance of open or closed conformation in equilibrium.

\section{Quasispecies Can Provide Adaptive Genetic Diversity of Viral Variants and Its Antigenic Determinants, Helping to Evade Immunosurveillance}

When a new host is infected, the infection actually occurs not with one virus variant, but with a whole population of genetically related but diverse variants that arise as a result of mutations during the replication of the virus in the previous hosts [37]. This population of virus variants is called quasispecies. The concept of quasispecies in relations to viral variants is very 


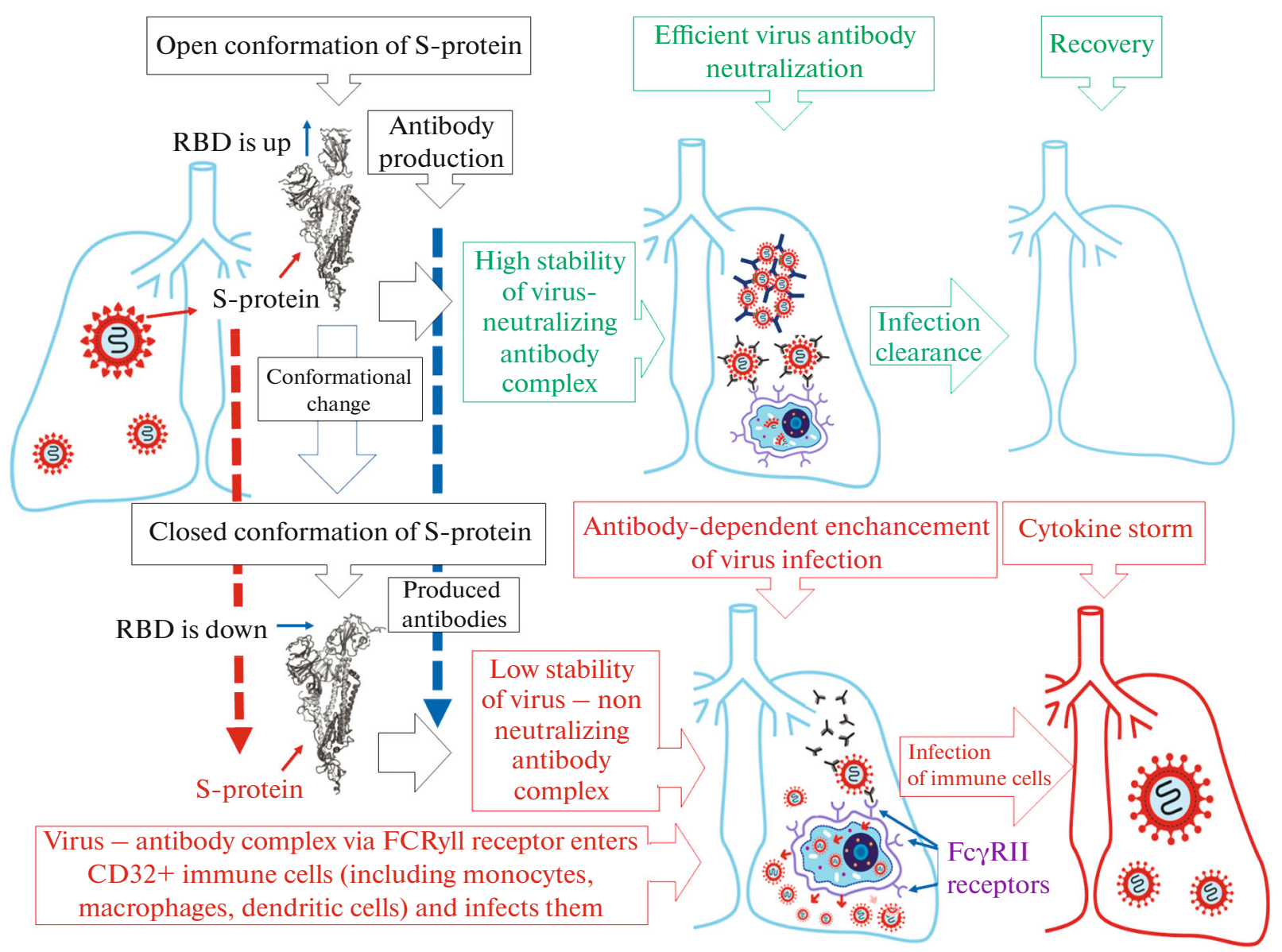

This graphic illustration was designed and created by Olga Zolotuchina, Olga Matveeva and Yurii Nechipurenko

Fig. 3. Schematic representation of possible relationship between conformation change of viral S-protein and ADE promoted severe form of COVID-19.

important because it helps to understand that in order to escape from the immunity of a new viral host by changing antigenic determinants, the virus does not have to acquire new mutations, it can take advantage of existing ones that occurred during replication in previous hosts. For example, some variants of viral quasispecies can have predominantly open S-conformation and therefore can be easily neutralized by antibodies of the new host, while other variants of the virus with closed conformation and worse affinity for antibodies can gain an evolutionary advantage after neutralization failure that can lead to infection of immune cells.

\section{A Possible Explanation for the More Frequent Occurrence of a Severe Form of the Disease in the Elderly People}

There are several hypotheses associated with the ADE phenomenon that explain the more severe course of the COVID-19 disease in the elderly people. The production of antibodies in older people is slower than in younger. Perhaps by the time the neutralizing antibodies have developed to the virus with the open S-protein conformation, the virus with the closed conformation starts to prevail. However, neutralizing antibodies to the open conformation of the S-protein, having reached a high concentration, can start forming unstable complexes with the virus with closed conformation. Moreover, they can "drag in" the virus into monocytes or macrophages, where the virus will be able to replicate. This process may be accompanied by a generalized development of infection and a cytokine storm that can be observed in some COVID-19 patients.

This hypothesis is supported by the detection of correlation between the titer of antibodies targeting RBD in the serum of COVID-19 patients and the disease severity: the higher the titer, the worse the disease [38]. The titer of antibodies targeting S-protein also correlated with age. Higher titers were found in older patients. In addition, a positive and significant correlation was found between these antibodies titer and the concentration of the C-reactive protein, which is an inflammation marker [39]. 


\section{Hypothetical Relationship between Antigenic Drift of SARS-CoV-2 and ADE}

ADE may happen due to antigenic drift of S-protein that occurs as a result of mutations of the gene encoding this viral protein $[29,40]$. These mutations can cause a change in both conformational and linear antigenic determinants. A thorough analysis of the variability of amino acids in different proteins of the SARS-CoV-2 virus in a database that included other betacoronaviruses demonstrated a relationship between the variability of amino acids and their position in antibody accessible parts of the S-protein molecule. In particular the S1 subunit, that includes the $\mathrm{RBD}$ domain and exposed in the virion, has a greater number of variable amino acids compared to less exposed S2 subunit [24, 41]. Structural proteins that are hidden inside the virion, or nonstructural proteins, are more conservative [24, 41]. The authors of study explain this variability by antigenic drift. The virus changes amino acids to change antigenic determinants and escape from neutralizing antibodies. Such an escape process, hypothetically, may be accompanied by ADE.

It is possible that 22 glycosylation sites in S-protein contribute to the antigenic diversity of the virus [42]. The idea that amino acid substitutions, conformational changes of S-protein, and a change in glycosylation pattern help the virus to avoid immune surveillance and promote ADE is not new. It was formulated in earlier publications (see [35, 41]).

What is the ratio between completely open, closed, or intermediate conformations of the S-protein in COVID-19 patients? What factors affect the equilibrium? Perhaps amino acid substitutions in certain positions of the S-protein, as mentioned above, can shift the equilibrium, so that a completely open conformation becomes thermodynamically favorable and predominates. It has been shown that some mutations of the SARS-CoV-1 virus genome can stabilize a certain conformation of the S-protein [27]. Perhaps the opposite scenario is also possible. Namely, some amino acid substitutions in certain positions of the Sprotein shift the equilibrium, making the closed conformation of the S-protein thermodynamically favorable and predominant. Theoretically, such substitutions can promote ADE.

Most likely the virus with the prevailing closed conformation of S-protein due to its low affinity for the ACE2 receptor will be less infectious for its host target cells. However, such a virus can gain some evolutional advantage via infection of immune cells using ADE strategy.

\section{CONCLUSIONS FOR ANTIVIRUS VACCINE DESIGN}

If our hypothesis that the diversity of the SARS$\mathrm{CoV}-2$ virus $\mathrm{S}$-protein antigenic determinants can cause an antibody-dependent enhancement (ADE) of infection and, as a result, the COVID-19 disease complications is true, then it can help in directing vaccine research.

According to our hypothesis, which we also formulated earlier [43], ADE is more likely to occur when a person vaccinated with a virus or genetic construct expressing an S-protein with a predominant open form of RBD, is getting infected with a virus with a predominant closed conformation of this protein. Since the affinity of antibodies to antigenic epitopes of the $\mathrm{S} 1$ subunit and RBD domain can change along with the conformation of the S-protein's change, we believe that antibodies to these epitopes are more likely to trigger ADE. Therefore, a vaccine targeting these epitopes in the $\mathrm{S} 1$ subunit is more likely to trigger the production of antibodies that can induce ADE following natural viral infection. An immune response to the S2 subunit of S-protein or to other conserved antigenic determinants of viral proteins is less likely to contribute to the ADE. Consequently, the vaccine that target these epitopes in S1 subunit more likely to trigger the production of antibodies that can cause ADE after natural virus infection. Immune response to the S2 subunit of the S-protein or other conservative antigenic determinants of structural or nonstructural viral proteins is less likely to promote ADE. Perhaps, proteins with these determinants can be used as antigens to stimulate the immune response during development of next-generation vaccines.

\section{ACKNOWLEDGMENTS}

The authors are grateful to A.N. Vzorov, A.A. Adzhubei, D.V. Kuprash, N.G. Esipova and M.V. Supotnitsky for useful discussions.

\section{FUNDING}

This work was supported by the Fundamental Research Program of the State Academies of Sciences for 2013-2020 (topic no. 01201363818) and the Russian Foundation for Basic Research, project no. 20-04-01085.

\section{REFERENCES}

1. J. Shang, Yu. Wan, Ch. Luo, et al., Proc. Natl. Acad. Sci. U.S.A. 117 (21), 11727 (2020)

2. M. S. Yip, H. L. Leung, P. H. Li, et al., Hong Kong Med. J. 22 (3-4), 25 (2016).

3. Q. Wang, L. Zhang, K. Kuwahara, et al., ACS Infect. Dis. 2 (5), 361 (2016).

4. L. Liu, Q. Wei, Q. Lin, et al., JCI Insight 4 (4), e123158 (2019).

5. S. M. Tirado and K. J. Yoon, Viral Immunol. 16 (1), 69 (2003).

6. M. Jaume, M. S. Yip, Y. W. Kam, et al., Hong Kong Med J. 18 (2), 31-36 (2012). 
7. M. S. Yip, N. H. Leung, C. Y. Cheung, et al., Virol. J. 11, 82 (2014).

8. Y. Wan, J. Shang, S. Sun, et al., J. Virol. 94, e02015 (2020).

9. Y. W. Kam, F. Kien, A. Roberts, et al. Vaccine 25, 729 (2007). L. Li, J. Wo, J. Shao, et al., Virus Res. 107 (1), 93 (2005).

10. K. Murphy and C. Weaver, Janeway's Immunobiology (Garland Science, New York, NY, 2016).

11. A. Banerjee, J. A. Nasir, P. Budylowski, et al., Emerg. Infect. Dis. 26, 2054 (2020).

12. M. C. Pontelli, I. A. Castro, R. B. Martins, et al., Preprint at bioRxiv (2020). https://doi.org/10.1101/2020.07.28.225912

13. A. Taylor, S. S. Foo, R. Bruzzone, et al., Immunol. Rev. 268, 340 (2015).

14. A. Takada and Y. Kawaoka, Rev. Med. Virol. 13 (6), 387 (2003).

15. M. Merad and J. C. Martin, Nat. Rev. Immunol. 20, 355 (2020).

16. J. A. Tetro, Microbes Infect. 22 (2), 72 (2020).

17. A. Iwasaki and Y. Yang, Nat. Rev. Immunol. 20, 339 (2020).

18. B. Tripet, M. W. Howard, M. Jobling, et al., J. Biol. Chem. 279 (20), 20836 (2004).

19. A. C. Walls, M. A. Tortorici, J. Snijder, et al., Proc. Natl. Acad. Sci. U.S.A. 114 (42), 11157 (2017).

20. J. Ou; Z. Zhou, R. Dai, et al., BioRxiv (2020). https://doi.org/10.1101/2020.03.15.991844

21. M. Yuan, N. C. Wu, X. Zhu, et al., Science 368, 630 (2020).

https://doi.org/10.1126/science.abb7269

22. F. Li, M. Berardi, W. Li, et al., J. Virol. 80 (14), 6794 (2006).

23. S. Matsuyama and F. Taguchi, J. Virol. 83 (21), 11133 (2009).

24. A. C. Walls, Y. J. Park, M. A. Tortorici, et al., Cell 181 (2), 281 (2020).

25. D. Wrapp, N. Wang, K. S. Corbett, et al., Science 367 (6483), 1260 (2020).
26. Y. Jia, G. Shen, Yu. Zhang, et al., BioRxiv (2020). https://doi.org/10.1101/2020.04.09.034942

27. R. N. Kirchdoerfer, N. Wang, J. Pallesen, et al., Sci. Rep. 8 (1), 15701 (2018).

28. M. Hoffmann, H. Kleine-Weber, S. Schroeder, et al. Cell 181, 271 e278 (2020).

29. Z. Y. Yang; H. C. Werner; W. P. Kong, et al., Proc. Natl. Acad. Sci. U.S.A. 102, 797 (2005).

30. A. Merk and S. Subramaniam, Curr. Opin. Struct. Biol. 23 (2), 268 (2013).

31. A. Bartesaghi, A. Merk, M. J. Borgnia, et al., Nat. Struct. Mol. Biol. 20 (12), 1352 (2013).

32. J. B. Munro, J. Gorman, X. Ma, et al., Science 346 (6210), 759 (2014).

33. B. Z. Zhang, Y. F. Hu, L. L. Chen, et al., Cell Res. 30, 702 (2020).

34. H. Wang, X. Hou, X. Wu, et al., Nat. Commun. 11, 3581 (2020).

35. B. Korber, W. M. Fischer, S. Gnanakaran, et al., Cell 182 (4), 812 (2020).

36. A. A. Adzhubei and M. J. Sternberg, J. Mol. Biol. 229 (2), 472 (1993).

37. I. N. Lu, C. P. Muller, and F. Q. He, Virus Res. 283, 197963 (2020). https://doi.org/10.1016/j.virusres.2020.197963

38. J. Zhao, Q. Yuan, H. Wang, et al., Clin. Infect. Dis. (2020). https://doi.org/10.1093/cid/ciaa344

39. F. Wu, A. Wang, M. Liu, et al., MedRxiv (2020). https://doi.org/10.1101/2020.03.30.20047365

40. H. Yao, X. Lu, Q. Chen, et al., MedRxiv (2020). https://doi.org/10.1101/2020.04.14.20060160

41. D. O. Ricke and R. W. Malone, Preprints with the Lancet (2020). https://doi.org/10.2139/ssrn.3546070

42. Y. Watanabe, J.D. Allen, D. Wrapp, et al., Science, eabb9983 (2020)

43. T. A. Zaichuk, Y. D. Nechipurenko, et al., Mol. Biol. 54, (2020). https://doi.org/10.1134/S0026893320060151 